\title{
Commission santé CNFG : journée jeunes chercheurs 2016
}

\section{(2) OpenEdition}

Journals

Édition électronique

URL : http://journals.openedition.org/rfst/608

DOI : 10.4000/rfst.608

ISSN : 2492-3672

Éditeur

Espaces et SOciétés (UMR 6590)

Référence électronique

"Commission santé CNFG : journée jeunes chercheurs 2016 », Revue francophone sur la santé et les territoires [En ligne], Ateliers et séminaires, mis en ligne le 25 novembre 2016, consulté le 06 avril 2021. URL : http://journals.openedition.org/rfst/608 ; DOI : https://doi.org/10.4000/rfst.608

\section{c.) (i) (2)}

La Revue francophone sur la santé et les territoires est mise à disposition selon les termes de la Licence Creative Commons Attribution - Pas d'Utilisation Commerciale - Partage dans les Mêmes Conditions 4.0 International. 
COMMISSION SANTE CNFG : JOURNEE JEUNES CHERCHEURS 2016.

Reprenant la pratique de ses prédécesseurs, la nouvelle commission santé du CNFG (http://www.cnfg.fr/) a souhaité organiser une journée de rencontres consacrée aux travaux des jeunes chercheurs. La réunion s'est tenue le vendredi $25 / 11 / 2016$ de $13 \mathrm{~h}$ à $17 \mathrm{~h}$, à I'Université de Rouen, Maison de l'Université sur le campus de Mont Saint Aignan. Le secrétariat scientifique a été assuré par deux doctorants : Renaud Misslin et Elise Autrive .L'objectif a consisté à proposer une tribune pour partager et discuter les innovations et la variété des approches de la sous discipline; une bonne manière de mettre en avant la pluralité des « géographies de la santé » (A. Gatrell, 2002).

L'idée serait désormais d'instaurer I'annualisation de cette tradition : une journée jeunes chercheurs chaque dernier vendredi de novembre, seuls les lieux pouvant changer. Cette année, la première partie de la réunion a été consacrée à une conférence de Éric Daudé (Chargé de Recherches CNRS, UMR IDEES, Rouen). Avec beaucoup de pédagogie, il a expliqué en quoi son travail issu des travaux sur la complexité pouvait croiser les préoccupations sanitaires. En particulier il a montré comment il devenait possible d'engager des simulations sur plate-forme informatique, de façon à mieux comprendre et lutter contre la diffusion des épidémies dans les métropoles du monde.

La seconde partie a vu se succéder 5 présentations par des doctorants de diverses universités, quatre sont présentées dans ce dossier :
Thorigny Maxime (Univ. Reims, REGARS EA 6292) : Le développement de la télémédecine en France. Une territorialisation de la politique publique ? Discutante : Lucas Véronique

Georgelin Béatrice (Univ. Angers, ESO 6590) : Les processus de territorialisation à l'œuvre dans les politiques de santé locales : l'exemple de Paris. Discutante : Autrive Elise

Lucaccioni Heloïse (Univ. Paris Ouest Nanterre, LADYSS 7533) : Une géographie des vulnérabilités territoriales face aux risques zoonotiques émergents. Discutant : Thorigny Maxime

Misslin Renaud (Univ. Rouen, UMR IDEES 6266) : Génération d'environnements pour la simulation à base d'agents : le milieu d'Aedes aegypti à Bangkok (Thaïlande). Discutante : Lucaccioni Heloïse

Chaque intervention a été suivie d'une série de questions/remarques, préalablement préparées par un des participants. Cette méthode a donné lieu à des échanges soutenus. Ils se sont prolongés pendant les pauses, offertes par le laboratoire CNRS/IDEES Rouen.

Pour garder une trace de ces moments et les valoriser, la revue Francophone sur la Santé et les Territoires (http://rfst.hypotheses.org/) a aimablement proposé de diffuser les résumés dans son espace séminaires/ateliers. Tous nos remerciements à son rédacteur en chef Sébastien Fleuret, pour son soutien actif.

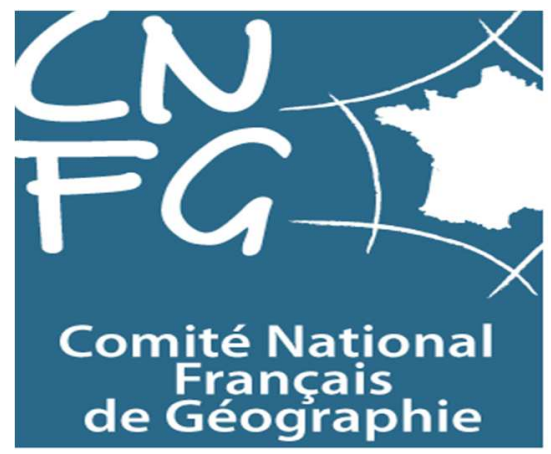




\section{LES SYSTEMES PATHOGENES COMPLEXES. MODELISATION ET SIMULATION}

\section{Éric DAUDÉ \\ CNRS, UMR IDEES \\ eric.daude@cnrs.fr}

Dans la lignée des travaux des géographes sur les complexes pathogènes (M. Sorre, 1933) et les systèmes pathogènes ( $\mathrm{H}$. Picheral, 1983), nous présentons ici quelques exemples destinés à promouvoir le concept de système pathogène complexe. En effet, dans le vocabulaire des sciences de la complexité, les risques pour la santé résultent moins d'une succession d'évènements que d'une articulation complexe de facteurs (Daudé, Vaguet \& Paul, 2015).

Prenons l'épidémie de grippe de 2017: La surmortalité qui sera probablement mesurée en France est-elle dû à une réduction de la couverture vaccinale? À un vaccin moins efficace ou une grippe plus virulente ? À une médecine de ville et des hôpitaux publics surchargés? À des conditions climatiques exceptionnelles ? La réponse est ardue car les épidémies s'inscrivent dans des systèmes sociospatiaux hétérogènes, constitués de nombreux niveaux décisionnels aux motivations et priorités diverses. Il en va ainsi des composants et comportements individuels, influencés par les structures familiales et les normes sociales, elles-mêmes rythmées selon les politiques de santé publiques et les contextes sociaux, qui sont en partie le reflet des forces du marché et des changements environnementaux. Ces systèmes comportent donc de nombreux éléments qui ont leurs propres dynamiques, sont évolutifs et en interactions permanentes. Ceux-ci produisent cette variabilité sociale et géographique de l'expression des épidémies, que l'on ne saurait réduire à de simples causalités. Pris ensemble, ces différents composants sont les termes classiques des systèmes pathogènes complexes. De tels systèmes possèdent des propriétés qui peuvent constituer des opportunités d'intervention en santé publique.

\section{Le charme discret des systèmes complexes}

Un système pathogène complexe est un système ouvert. C'est ce que l'on observe lorsque le virus Ébola sort de son aire d'endémie via les mobilités des populations (Gomes et al.,
2014), ou lorsque des pratiques culturelles se « propagent »: I'alimentation fast-food, et induisent de nouveaux risques, l'obésité (De Vogli et al., 2014). Si cette ouverture du système accroit le risque de diffusion de pathogènes ou de pratiques à risque, elle oblige également à une meilleure coopération internationale en matière de santé, et notamment de surveillance et de prévention.

Un système pathogène complexe présente des dynamiques non-linéaires. Dans les maladies à vecteurs (paludisme, dengue, Zika...), les densités vectorielles sont certes liées aux conditions climatiques mais aussi aux politiques de contrôle et aux variations spatiales des gites de ponte (Reiter, 2008). De petits évènements volontaires (destructions ciblées de gites) ou des variations incontrôlées (climatique) pourraient alors générer, par effet systémique, de grand effets (réduction ou extension des aires de présence du vecteur). La recherche de ces seuils et «petits évènements » à l'origine de grands impacts devrait devenir un objectif prioritaire dans la lutte contre les épidémies.

Un système pathogène complexe est caractérisé par une variabilité spatio-temporelle de l'information. L'omniprésence des flux d'information dans ces systèmes assure la réactivité de ses éléments face aux changements: la diffusion des pratiques de prévention a été un moyen de lutte efficace contre le sida (Hornik, 2002). Cette propriété peut donc être mise au service de la santé publique. C'est le cas par exemple des études sur les mots-clés (liés à la grippe, au choléra etc.) des requêtes sur Internet ou dans les réseaux sociaux qui servent à suivre la progression d'une maladie dans des pays où le système de surveillance est défaillant (Chunara et al., 2012). Mais ces flux peuvent être sources d'influence interdépendantes et contradictoires : amalgames et contre-vérités scientifiques, crédibilités des opérateurs publics et lobbying de l'industrie pharmaceutique seraient à confronter aux évolutions de la couverture vaccinale. 
Un système pathogène complexe connaît des processus d'auto-organisation. Ces processus décentralisés génèrent spontanément des propriétés nouvelles et organisées, sans I'intervention d'une autorité supérieure. Ces processus sont à l'origine de l'émergence des patients-experts du fait de changements dans la relation au corps et de l'avènement de la société de l'information (Boudier et al., 2012). Ces phénomènes émergents, ou innovations sociales, peuvent parfois être « récupérés » par le système pour s'imposer à l'ensemble, notamment s'ils engendrent de meilleures performances ou s'ils permettent une réduction des dépenses publiques (Donaldson, 2003).

Un système pathogène complexe présente une sensibilité aux conditions initiales, son évolution est en partie liée à son histoire. Les états, expériences et expositions passés ont donc une part importante dans la dynamique à venir du système. La séroprévalence est un exemple classique de cette sensibilité, la propagation d'un virus dans une population étant en partie liée à ses précédents passages et au taux d'immunité acquise par la population (Ooi et al., 2006).

Enfin un système pathogène complexe est toujours loin de l'équilibre, en équilibre instable. Si l'équilibre est considéré dans le cas des maladies vectorielles non pas comme l'absence de la maladie mais comme la circulation du virus à bas bruit, alors cet équilibre peut basculer vers des bouffées épidémiques, voire des pandémies, du fait des propriétés dont nous venons de parler: ouverture, interactions locales et globales, dynamiques non-linéaires, sensibilité aux conditions initiales. Les allergies ou les déficiences immunitaires sont d'autres exemples, à l'échelle de l'individu, de l'équilibre instable du système immunitaire.

\section{Apprendre en modélisant à base d'agents}

$\mathrm{Si}$ ces concepts issus des sciences de la complexité sont élégants, pour mieux caractériser une définition déjà ancienne d'un système pathogène (Picheral, 1983 ; Handschumacher \& Hervouët, 2004); nos pratiques de recherche nous conduisent à les formaliser par des modèles et à explorer leurs dynamiques par la simulation informatique, apportant ainsi l'élément manquant au système pathogène complexe (Gatrell, 2005). La modélisation à base d'agents constitue le cadre méthodologique employé. Techniquement il s'agit de systèmes informatiques composés d'entités appelées agents, qui interagissent mutuellement ou avec leur environnement, qui possèdent le plus souvent un but ou un objectif à atteindre et qui procèdent selon des modes de coopération, de concurrence ou de coexistence. Ce qu'il faut retenir ici c'est la souplesse de ce type de modélisation algorithmique, basé sur des propriétés et des méthodes appliquées à des agents, par rapport à une modélisation mathématique basé sur des fonctions et des processus appliquées à des variables.

Les modèles à base d'agents permettent en effet d'intégrer dans un même système une grande variété de catégories qui agissent et une forte hétérogénéité entre les individus de ces catégories. On peut ainsi modéliser des hôtes, des vecteurs et un système de soins, avec des différenciations sociodémographiques, génétiques et géographiques. La prise en compte de cette diversité interindividuelle peut s'avérer intéressante dans la recherche d'effets macroscopiques d'interventions réalisées sur des populations cibles.

Les modèles à base d'agents offrent également la possibilité de formaliser explicitement des comportements, comme les mobilités individuelles, et des mécanismes décisionnels, comme le choix d'une pratique de prévention individuelle. Le choix de tel ou tel comportement peut s'appuyer sur des informations qui sont inégalement distribuées dans l'espace, le temps et entre agents. Ces comportements peuvent par ailleurs être contradictoires ou évolutifs selon les informations reçues et ce faisant être plus proche des pratiques observées, telles que les dynamiques d'opinion par exemple.

Les modèles à base d'agents donnent enfin à l'espace un statut de composant du système modélisé. Il peut donc être décrit comme un agent, avec les hétérogénéités et les dynamiques géographiques et environnementales jugées nécessaires à l'évolution du système. La construction d'un modèle épidémiologique à l'échelle d'une ville intégrera ainsi les modes d'utilisation du sol, les densités de population ou encore la localisation des hôpitaux, en général toute information spatiale supposée agir dans le système ainsi décrit. 


\section{Le cas exemplatif de M03}

Illustrons maintenant ces concepts et options méthodologiques avec le projet $\mathrm{MO}^{3}$. Les maladies à transmissions vectorielles (MTV) sont un enjeu majeur de santé publique avec un fort potentiel d'explosions épidémiques, au regard notamment du changement climatique et des dynamiques d'urbanisation. La surveillance et le contrôle de ces MTV passent notamment par la production de bases de données géographiques et de modèles dynamiques. Ceux-ci peuvent contribuer à l'évaluation des risques vectoriels et des risques épidémiques à différentes échelles, notamment micro-locale des espaces urbains. Dans ce contexte, $\mathrm{MO}^{3}$ est un simulateur de maladies à transmission vectorielle dont l'objectif est d'explorer la capacité de reproduction et de dispersion du vecteur Aedes aegypti selon différentes configurations géographiques et le rôle des mobilités humaines dans la dispersion des virus aux échelles intra-urbaine.

$\mathrm{MO}^{3}$ est un modèle à base d'agents où sont décrits les quatre principaux sous-système de la dengue: les humains (Cebeillac \& Daudé, 2016), les moustiques (Maneerat \& Daudé, 2016), I'environnement (Misslin \& Daudé, 2016) et les virus. Bangkok (Thaïlande) et Delhi (Inde) sont les métropoles d'étude sélectionnées pour calibrer ces différents composants.

Tout d'abord la population d'hôtes est générée informatiquement de façon à respecter statistiquement le genre et la pyramide des âges, observée dans la réalité à une échelle fine. Pour chaque agent des attributs concernant le statut immunitaire sont créés, en relation avec la base de données sur les virus, afin de suivre la progression de la maladie. Des données issues de l'analyse de réseaux sociaux (Twitter) permettent de calibrer une partie des agendas des mobilités individuelles. Dans le jargon de la modélisation agents, l'ensemble de cette phase consiste à construire une population synthétique, socialement et spatialement représentative de la population cible.

Le vecteur est modélisé à l'échelle de l'individu pour les adultes et sous la forme de stocks de moustiques pour les stades aquatiques. Si le formalisme employé pour modéliser les stocks et leurs transitions repose sur des fonctions mathématiques, la modélisation des comportements du moustique individualisé s'appuie sur les connaissances entomologiques, notamment en termes de prédation, de ponte et de conquête territoriale.

Enfin l'environnement est défini à la fois selon les besoins et les contraintes liées au cycle de vie du moustique et aux activités humaines. Le modèle environnemental intègre donc des caractéristiques hétérogènes dans le type d'espace (usages du sol), dans le temps (variations climatiques) et dans les comportements (contrôle différencié des gites de pontes).

Le couplage de ces trois sous-systèmes avec un quatrième module représentant une base de connaissance sur les virus constitue donc le modèle $\mathrm{MO}^{3}$. La construction de ce modèle participe donc d'une part à une démarche heuristique de formalisation des connaissances autour d'un système pathogène complexe et d'autre part à un besoin d'explorer des dynamiques endémies-épidémies selon différents scénarii. Ainsi les villes « synthétiques » seront destinées à accueillir des exercices de simulations d'hypothèses de recherche et à mesurer des dynamiques qu'il n'est pas possible d'observer dans la réalité, tels que les lieux de contamination par les moustiques.

Concluons cette présentation rapide en apportant quelques réponses à des critiques qui sont régulièrement adressées à l'encontre de cette catégorie de modèles à base d'agents (comportemental et individu-centré) par rapport à des modèles mathématiques (de fonction et agrégé).

La première critique porte sur l'intérêt limité, voire contre-productif, d'une modélisation à une échelle aussi fine, là où des propriétés agrégées suffisent à caractériser des dynamiques macroscopiques. Signalons que de telles propriétés macroscopiques ont pour la plupart été révélées par l'observation directe ou par une multitude d'expérimentations, qui sont extrêmement compliquées à mettre en œuvre en épidémiologie spatiale, ou qui, lorsqu'elles existent, sont sujettes aux nombreux biais connus des données de surveillance. En partant des comportements, du moustique et de l'hôte par exemple, on intègre des connaissances qui reposent là aussi sur de nombreuses études (déplacement, espace de vie, prédation) pour ensuite produire, par la simulation, des 
connaissances macroscopiques qui peuvent non seulement converger vers des observations faites par ailleurs (par exemple sur la capacité de dispersion de cohortes de moustiques) mais aussi vers des connaissances nouvelles difficiles à observer sur le terrain (graphe des cycles de contamination hôtes-vecteurs par exemple). Le fait de prendre en compte la diversité des types d'agents, leur hétérogénéité et leurs comportements permet ainsi l'exploration d'une très grande variété de dynamiques.

La seconde critique, liée en partie à la première, porte sur la difficulté, voire l'impossibilité, de calibrer, de valider et d'interpréter les résultats issus des simulations de modèles intégrant de nombreux composants et interactions nonlinéaires. C'est effectivement un verrou que tout modélisateur prend en compte lorsqu'il réalise ce type de modèle dans lequel il peut y avoir des dizaines voire des centaines de paramètres. Tout d'abord, la calibration du modèle repose pour l'essentiel sur les connaissances dans les différents domaines d'exercice du modèle (en entomologie par exemple). Chaque valeur de paramètre doit donc reposer sur des données dont les plages de variation sont connues afin de réduire au maximum l'incertitude. Ensuite on dispose aujourd'hui de méthodes statistiques liés à l'explosion des big-data qui permettent de traiter et d'analyser des volumes de données tels que ceux en sortie de simulations. Enfin il reste la question de la validation, qu'il faut « désacraliser » par rapport aux pratiques de validation classique. Il est en effet toujours possible, même si le nombre de paramètre libre n'est pas très grand, de faire converger les simulations vers des valeurs empiriques observées. Il faut donc d'une part s'assurer que cette convergence se réalise dans le respect des plages de variations connues des paramètres des entités élémentaires. Il faut d'autre part discuter avec les experts du domaine des diverses dynamiques du modèle. Certes une même situation peut-être reproduite à partir de différentes combinaisons de paramètres, mais n'est-ce pas là une possibilité du monde réel ! Il faut enfin mettre à la disposition de tous ces modèles et jeux de données pour faciliter la reproductibilité des simulations.

Pour conclure, il y a donc des avantages à construire des modèles à base d'agents, spatialisés et explicites, pour l'épidémiologie des systèmes pathogènes complexes. Les hypothèses à l'origine de chacun des comportements modélisés et de leurs conditions de mise en œuvre sont aisément testables et discutables. Leur modélisation et l'étude de leurs dynamiques durant la simulation permettent de mesurer leur contribution dans l'évolution du système, et éventuellement de les supprimer si le système se comporte de manière aberrante par rapport à nos connaissances. Plutôt que de modéliser des processus définis à partir d'observations macroscopiques, la modélisation de comportements microscopiques peut permettre de faire émerger des comportements surprenants. Ceux-ci poseront de nouvelles questions et orienteront vers des études de terrain, pour confirmer ou infirmer ces solutions. En matière de modélisation et de simulation à base d'agents en épidémiologie spatiale, je prônerai donc de l'ambition pour les programmes de recherche et de la modestie dans les conclusions tirées des résultats des simulations.

\section{Bibliographie}

Boudier F., Bensebaa F., Jablanczy A. (2012), L'émergence du patient-expert: une perturbation innovante. Innovations, 3, 39:1325.

Cebeillac A., Daudé É. (2016), Où et Quand? Que nous apprennent les données Twitter et Google Map sur les mobilités quotidiennes de Bangkok (Thailande). 15e coll. Mobilités Spatiales, Fluidité Sociale (MSFS), Champs-surMarne, https://msfs2016.sciencesconf.org/111848

Chunara R., Andrews J., Brownstein J. (2012), Social and News Media Enable Estimation of Epidemiological Patterns Early in the 2010 Haitian Cholera Outbreak. The American Society of Tropical Medicine and Hygiene, 86, 1: 39-45.

Daudé É., Vaguet A., Paul R. (2015), La dengue, maladie complexe. Natures Sciences Sociétés, 23, 4: 331-342, 10.1051/nss/2015058

De Vogli R., Kouvonen A., Gimeno D. (2014), The influence of market deregulation on fast food consumption and body mass index: a cross-national time series analysis. Bulletin of World Health Organisation, 92:99-107.

Donaldson L. (2003), Expert patients usher in a new era of opportunity for the NHS. British Medical Journal, 326, 7402: 1279-1280. 
Gatrell A. (2005), Complexity theory and geographies of health: a critical assessment. Social Science \& Medicine, 60;12:2661-71.

Gomes M., Pastore y Piontti A., Rossi L., Chao D., Longini I., Halloran M., Vespignani A. (2014), Assessing the International Spreading Risk Associated with the 2014 West African Ebola Outbreak. PLOS Currents Outbreaks.

Handschumacher P., Hervouët J.-P. (2004), Des systèmes pathogènes à la santé publique : une nouvelle dimension pour la géographie de la santé tropicale, Autrepart, 1;29:47-63.

Hornik R. (Ed.). (2002), Public health communication: Evidence for behavior change. Routledge, London, p. 421.

Maneerat S., Daudé É. (2016), A spatial agentbased simulation model of the dengue vector Aedes aegypti to explore its population dynamics in urban areas. Ecological Modelling, 333:66-78, 10.1016/j.ecolmodel.2016.04.012.
Misslin R., Daudé É. (2016), Génération d'environnements artificiels pour la simulation spatiale d'arboviroses en milieu urbain : application à la dengue et au virus Zika. 16e coll. SAGEO, Nice, https://sageo2016.sciencesconf.org/

Ooi E., Goh K., Gubler D. (2006), Dengue prevention and 35 years of vector control in Singapore. Emerging Infectious Diseases, 12;6:887-93.

Picheral H. (1983), Complexes et systèmes pathogènes : approche géographique, De l'épidémiologie à la géographie humaine. Travaux et documents de géographie tropicale, n० 48, p. 5-22.

Reiter P. (2008), Global warming and malaria: knowing the horse before hitching the cart. Malaria Journal, 7:Suppl 1, S3. 


\title{
LE DEVELOPPEMENT DE LA TELEMEDECINE EN FRANCE. UNE TERRITORIALISATION DE LA POLITIQUE PUBLIQUE ?
}

\author{
Maxime THORIGNY \\ REGARS - EA 6292, Université de Reims Champagne-Ardenne \\ maxime.thorigny@gmail.com
}

Le développement de la télémédecine en France est consacré par la loi Hôpital, Patient, Santé Territoires (HPST) de 2009, son décret d'application de 2010 définit 5 types d'actes, permettant de réaliser des actes médicaux au moyen «d'un dispositif utilisant les technologies de l'information et de la communication ». La principale innovation réside donc dans un changement organisationnel de la pratique médicale. Les objectifs affichés par les différents ministres de la santé sont clairs: dans un premier temps réduire les inégalités d'accès aux soins par une meilleure coordination à distance des professionnels, dans un second temps maitriser les dépenses de santé en réduisant le transport de patients, en prodiguant le bon soin au bon moment.

De manière concomitante, la planification du secteur de la santé est attribuée aux Agences Régionales de Santé, structures qui sont créées elles aussi par la loi HPST. Elles sont chargées d'établir la politique régionale en matière de santé en formalisant un Programme Régional de Santé qui définit les objectifs à poursuivre, sur la base d'un constat partagé. Différents territoires d'action sont envisagés, leur taille et leur mode de construction sont laissés libres pour chaque ARS. Le Programme Régional de Santé se décline en «schémas» et «programmes » dont le Programme Régional de Télémédecine (PRT), qui définit la politique à tenir en matière de télémédecine. Ce PRT est construit au niveau régional, sur la base d'un guide édité par la Direction Générale de l'Offre de Soins (DGOS) qui fixe la méthodologie et les priorités nationales.

La mise en œuvre des projets va se heurter à plusieurs écueils. Pour le secteur libéral, le manque de clarté juridique sur les outils et les responsabilités des acteurs et le caractère non pérenne des financements sont un frein majeur. Concernant les établissements publics comme privés, la problématique n'est pas seulement financière mais se pose aussi en terme de conduite de changement d'un point de vue organisationnel. Enfin, les ARS ont fait des choix techniques différents, en matière de « plateformes de télémédecine » qui sont le support des échanges entre praticiens. Il n'y a pas de logique frontalière dans le cadre de projets extrarégionaux.

Afin d'établir une évaluation chiffrée sur l'efficience dans le secteur ambulatoire et avant une généralisation, le Ministère de la santé a retenu 9 régions en 2014 comme territoires expérimentateurs: Alsace, Basse-Normandie, Bourgogne, Centre - Val de Loire, HauteNormandie, Languedoc-Roussillon, Martinique, Pays de la Loire et Picardie.

Cette communication vise donc à analyser la manière dont le territoire est pris en compte et quelles tensions existent entre la politique de déploiement de la télémédecine et la manière dont les acteurs de terrain s'en saisissent.

Dans un premier temps cette communication revient sur I'histoire de la notion de territoire dans le secteur de la santé et la définition du territoire d'action pertinent pour l'Etat (Coldefy et Lucas-Gabrielli (2010 et 2012), Vigneron (2009) et Rican (2004) et Vaillant (2009)). La taille, ainsi que la définition du territoire sont très variables et dépendent de la configuration territoriale elle-même (surface, densité, réseau de communication, besoin et offre de soins). La loi HPST donne le pouvoir aux ARS de définir les territoires avec les outils qu'elles souhaitent, il apparait donc une diversité très importante dans les approches aussi bien en terme de conception que de caractéristiques (rapport de 1 à 14 en terme de population, rapport de 1 à 440 en terme de surface). D'un autre coté I'analyse du contenu des PRT en utilisant le guide de la Direction Générale de I'Offre de Soins du Ministère de la santé comme grille de lecture révèle que toutes les priorités et les recommandations n'ont pas été respectées et que l'on trouve des initiatives régionales. Nous nous interrogeons donc sur la prise en compte des territoires et la manière dont ils sont 
appréhendés dans la politique de télémédecine des ARS ? Il semble que le territoire soit considéré comme un réseau entre praticiens de premier et second recours de manière formelle, car c'est sur cette dimension que se développe les projets de télémédecine et non en matière d'adéquation entre une offre et un besoin de soins spatialement localisé.

Dans un second temps nous nous attachons à étudier les mécanismes de l'expérimentation article 36, par la lecture du New Public Management (Pyun (2013) et Boyer (1997)) et le caractère performatif du discours de la tutelle (Jany-Catrice 2012), en imposant un cadre réglementaire très strict avec une contractualisation croisée entre I'ARS, la Caisse Primaire d'Assurance Maladie et les professionnels de santé. De la même manière, le tarif des expérimentations est jugé insuffisant par les professionnels ainsi que le périmètre (plaies chroniques et complexes). Le ministère n'a pas laissé de marge de manœuvre aux ARS quant à l'adaptabilité de l'expérimentation aux besoins des territoires ou aux structures existantes à l'exception du choix des prestataires des solutions des « plateformes de télémédecine ». A ce titre, nous montrons qu'il $n^{\prime} y$ a pas de logique frontalière claire, car les choix techniques ont été faits par région sur le fondement de marché publics. Plusieurs industriels se partagent le marché au niveau national, il n'existe pas encore de norme opposable concernant les formats d'échanges de données ce qui posera dans le futur proche des problèmes d'interopérabilité des systèmes dans une perspective de déploiement national (figure 1). Nous arrivons ici à une conclusion paradoxale, la télémédecine a pour vocation à s'affranchir des contraintes de localisation des professionnels de santé, mais sa politique de déploiement amène à renforcer les frontières régionales en rendant difficile voire impossible la coopération interrégionale. A ce jour, cette expérimentation a donné lieu à moins de 100 actes facturés depuis 2014. Le ministère de la santé, conscient des problèmes rencontrés,

1 Il existe deux types d'Accident Vasculaire Cérébral. Les AVC ischémiques qui se présentent sous la forme de l'obstruction d'un vaisseau sanguin et qu'il faut thrombolyser afin de permettre une circulation sanguine correcte, ce procédé consiste en l'injection d'un médicament fluidifiant le sang. Le deuxième type d'AVC est I'AVC hémorragique qui propose donc des modifications majeures de l'expérimentation : élargissement à l'ensemble des Affections Longues Durées, nouveaux tarifs plus attractifs, évaluations réalisées par la Haute Autorité de Santé à partir des bases de données de l'assurance maladie et suppression de la contractualisation qui pose la question de la place des ARS dans le suivi de ces expérimentations.

Enfin, nous montrons quels sont les déploiements en cours des projets de télémédecine et comment ils répondent à des besoins en matière de santé des territoires, où la co-production entre les différents acteurs, établissements, professionnels et tutelle, fonctionne autour d'un cadre institutionnel stable. Nous prendrons deux exemples nationaux, le déploiement du téléAVC et de la téléradiologie.

Le téléAVC consiste à la consultation à distance d'un chirurgien neurologue préalable à la thrombolyse médicamenteuse ${ }^{1}$, l'enjeu est de proposer un diagnostic dans les $4 \mathrm{~h}$ après la survenue des symptômes. Des Unités NeuroVasculaires sont dédiées à ces urgences particulières, cependant leur répartition territoriale est inégale et l'accessibilité est très variable. Le but est qu'un centre d'urgence traditionnel accessible plus rapidement, puisse bénéficier de l'expertise d'une UNV sans déplacer le patient. Plusieurs types d'organisations définies par les professionnels eux-mêmes à deux niveaux, au niveau de chaque équipe, centre requérant et requis, mais aussi entre établissements. 5 types d'organisations ont été synthétisés par l'Agence Nationale d'Appui à la Performance des établissements sanitaires et sociaux (ANAP) permettent d'adapter le fonctionnement interne des établissements et les relations interétablissement (ANAP 2014).

De la même façon le déploiement de la téléradiologie s'est fait dans une logique de coopération entre plusieurs sites et plusieurs

nécessite une intervention chirurgicale. Si la décision est prise de thrombolyser un AVC hémorragique le traitement aggravera significativement I'hémorragie cérébrale, c'est pourquoi le protocole impose l'avis d'un neurologue avant toute opération. 
professions, en effet, il n'y a pas besoin de professionnels médicaux dans le centre requérant, un manipulateur d'électro-radiologie est suffisant. Le droit reconnait cette pratique par la division en forfaits des actes techniques et intellectuels qui a été construite à l'aide des sociétés savantes et des représentants syndicaux.

Dès lors, nous mettrons en évidence que la coordination (Batifoulier 1992, Béjan 1999) entre les acteurs passe par la définition d'une convention partagée dans le sens normatif de la théorie des conventions. Celle-ci ne peut être qu'une co-construction territorialement cohérente et ne peut donc pas être définie $e x$ ante par le référentiel politique.

Le développement de la télémédecine en France passe par deux vecteurs: I'expérimentation nationale dite «article 36 » qui porte sur 9 anciennes régions et la politique régionale déployée par les ARS, sur la base de recommandations du Ministère de la santé. Le déploiement sur le terrain est très variable d'une région à l'autre car les objectifs des ARS sont différents. De même la définition qu'elles font du territoire ne repose pas sur les mêmes normes, ce qui aboutit à des espaces de projets de tailles variables.

Nous montrons qu'il y a un problème de définition de la politique publique en santé : d'un côté la loi HPST consacre les ARS et la région comme territoire de définition de la politique de santé, de l'autre les objectifs sont cadrés par le Ministère de la santé, sans tenir compte des spécificités des territoires. A ce titre, la télémédecine est un bon exemple de conflit de représentations et d'objectifs entre l'échelon national et régional.

L'expérimentation nationale se révèle être pour le moment un échec par l'imposition de contrats et de modes d'organisation, ne laissant pas la possibilité aux acteurs de s'organiser entre eux à leur convenance, en respectant les organisations déjà mises en place. En ce sens l'imposition de contrats nationaux ne permet pas la co-construction et la définition d'une convention partagée entre acteurs. Alors qu'il existe des exemples d'utilisation de la télémédecine, notamment concernant le traitement de I'AVC en urgence ou plus généralement la téléradiologie, qui s'adaptent aux pratiques des professionnels de santé en respectant les réseaux de recours professionnels déjà établis.

On peut se demander si la tutelle n'est pas en train de changer sa manière de déployer la politique de télémédecine. En effet, la réforme des Groupements Hospitaliers de Territoire (GHT), qui organise les établissements en réseaux de recours afin d'organiser la gradation des soins, institutionnalise la télémédecine comme une nouvelle norme de coopération entre professionnels d'établissements d'un même GHT avec pour objectifs de réduire les coûts de transports et de personnels. On s'oriente donc vers une co-construction aussi bien en interne qu'en externe des établissements.

\section{Bibliographie}

ANAP. (2014), « La télémédecine en action : prise en charge de I'AVC - Support d'aide au déploiement régional ».

BATIFOULIER P. (1992), «Le rôle des conventions dans le système de santé », Sciences Sociales et Santé

BEJAN S. (1999), « De nouvelles théories en économie de la santé : fondements, oppositions et complémentarités, Politiques et management public

BOYER R, (1997), «Evolution des modèles productifs », Paris, CEPREMAP

COLDEFY M. et LUCAS-GABRIELLI V. (2010), «Les territoires de santé: des approches régionales variées de ce nouvel espace de planification », Pratiques et Organisation des Soins, CNAMTS

COLDEFY M. et LUCAS-GABRIELLI V. (2012), « Le territoire, un outil d'organisation des soins et des politiques de santé ? », Questions d'économie de la Santé, IRDES

JANY-CATRICE F. (2010), «La performance totale: nouvel esprit du capitalisme? », Villeneuve d'Ascq, Presses universitaires du Septentrion

PYUN H. (2013), « Hybridation du New public Management: cas de gestion des administrateurs territoriaux en France, Management \& Avenir 
RICAN S. et VAILLANT Z. (2009), « Territoires et santé : enjeux sanitaires de la territorialisation et enjeux territoriaux des politiques de santé », Sciences sociales et Santé
VIGNERON E. (2004), « Des territoires de santé pour quoi faire? » Soins Cadres 


\section{STRATEGIES ET DYNAMIQUES TERRITORIALES A PARIS DANS LE CHAMP DE LA SANTE DE LA MERE ET DU JEUNE ENFANT.}

\section{Béatrice GEORGELIN \\ ESO - CNRS UMR 6590, Université d'Angers \\ beatrice.georgelin@univ-angers.fr}

L'amélioration des parcours de santé des femmes durant leur grossesse et des jeunes enfants est un objectif général de santé publique mobilisant de nombreux acteurs, à la fois institutionnels (État, Agences Régionales de Santé, Conseils Départementaux), professionnels (maternités, services de Protection Maternelle et Infantile, professionnels de santé libéraux) ainsi que d'autres acteurs de terrain, notamment associatifs.

Il se décline notamment en un objectif de réduction des inégalités socio-territoriales dans l'accès et la qualité du suivi proposé aux femmes et aux familles, objectif politique partagé par l'ensemble des acteurs publics de l'offre de santé dans ce champ². Pour l'atteindre, l'enjeu ne consiste pas seulement à optimiser la prise en charge actuelle, mais de réfléchir à une évolution des modes d'actions et à l'articulation des interventions de ces multiples acteurs, au plus près des besoins locaux. L'étude des reconfigurations territoriales de ce système d'acteurs permet de mettre en lumière les tensions et les limites des dynamiques en cours en regard de l'objectif de réduction des inégalités.

À Paris, cet objectif se traduit par un effort porté sur l'amélioration de la prise en charge des femmes et des familles les plus vulnérables, qui n'ont accès qu'à l'offre publique ou associative, effort conduit par la collectivité parisienne, au titre des compétences de Protection Maternelle et Infantile (PMI) du Département de Paris, et de nombreux partenaires du soin, de la prévention et de l'accompagnement.

Cette communication vise ainsi à :

\footnotetext{
${ }^{2}$ Ce champ, désigné comme celui de la santé périnatale, ou de la santé maternelle et infantile, ou encore de la santé de la mère et du jeune enfant, recouvre différentes questions de santé liées à la grossesse (planification familiale, suivi des grossesses normales, prise
}

- Présenter les acteurs de ce champ à Paris, à travers leurs différentes missions, ressources, stratégies et territorialités (Vanier, 2009).

- Montrer la pluralité et la complémentarité des stratégies territoriales mises en œuvre pour répondre aux besoins des plus vulnérables.

- Interroger les effets et les fragilités de ce système d'acteurs, dans un contexte actuel de pressions budgétaires croissantes.

Plus largement, c'est l'ambiguitté de la réduction des inégalités de santé en tant qu'objectif qui est à interroger, dans la mesure où les dynamiques observées à Paris témoignent d'un enjeu plus fondamental : I'adaptation de l'offre de santé afin qu'elle soit réellement accessible à tous.

Matériaux et méthode. Les résultats présentés ici sont issus de recherches menées dans le cadre d'une thèse portant sur les processus de territorialisation à l'œuvre dans I'action publique locale en santé à Paris, qui s'appuie sur une observation participante dans différentes démarches de «démocratie sanitaire » portées par la Mairie de Paris, en 2015 et 2016 ainsi que sur des entretiens réalisés auprès d'acteurs en santé, principalement publics. Ont été mobilisés pour cette présentation :

- Des données de terrain produites lors de la participation aux débats publics et aux ateliers des États Généraux de la PMI de Paris, démarche de concertation organisée en 2015. En particulier, celles recueillies lors des réunions de I'atelier «La PMI active dans les territoires » auxquelles participaient des professionnels de la PMI de

en charge médicale des grossesses à risques), à l'accouchement, au suivi du développement et de la santé du jeune enfant de 0 à 3 ans. 
Paris et leurs partenaires: Ateliers Santé Ville parisiens (ASV), professionnels du social et médico-social, chercheurs, ...

- Des entretiens réalisés en 2015 et 2016 auprès de professionnels et d'administrateurs de la PMI de Paris; de professionnels de l'Agence Régionale de Santé (ARS) d'Ile-de-France en charge de la périnatalité ; du réseau de santé périnatale parisien ; d'acteurs associatifs ...

\section{Contexte sociodémographique et sanitaire}

Paris présente une natalité importante et les principaux indicateurs en santé périnatale y sont plutôt bons, proches des taux moyens régionaux et nationaux (Vincelet et al., 2016) ${ }^{3}$. Toutefois, des disparités territoriales infraparisiennes, parfois importantes, peuvent être observées. Pour le suivi des grossesses, on observe par exemple des variations importantes entre arrondissements sur certains indicateurs clés, comme la proportion de femmes n'ayant pas réalisé l'ensemble des échographies recommandées durant leur grossesse ou le pourcentage de femmes déclarant tardivement leur grossesse (DASES, 2015).

Les travaux issus de l'enquête nationale périnatale ont montré la persistance de disparités sociales et territoriales en France en ce qui concerne les parcours de santé des mères et des jeunes enfants, et in fine les indicateurs sanitaires liés (Blondel, Kermarrec, 2011). De nombreuses vulnérabilités sont à prendre en compte pour expliquer ces disparités : vulnérabilités affectives et personnelles, vulnérabilités liées aux multiples formes de précarité, aux conditions de vie, de travail et de logement, aux ressources familiales et amicales, aux parcours de vie de ces femmes, notamment aux parcours de migrations ... (Gasquet, 2013).

Les disparités observées à Paris participent ainsi plus largement d'inégalités socio-territoriales de santé. La féminisation de la précarité, en particulier de la grande exclusion, l'aggravation de la précarité et la forte proportion de familles

3 Environ 29000 bébés parisiens naissent chaque année depuis 2011, le taux de natalité étant plus élevé dans les arrondissements du nord et nord-est parisien. La proportion élevée de mères étrangères à Paris est également à noter : ces dernières années, en moyenne, 30\% étrangères, pour certaines migrantes, sont, parmi cet ensemble de vulnérabilités et d'inégalités, les problématiques particulièrement saillantes aux yeux des professionnels de terrain à Paris.

2. Missions et problématiques des différents acteurs de l'offre de santé

Différents acteurs publics assurent la prise en charge médicale, l'offre de prévention et d'accompagnement des femmes tout au long des parcours de grossesse, puis des enfants après leur naissance.

En comparaison d'autres départements d'Ilede-France, où peuvent être observés des problèmes de couverture territoriale, cette offre de santé est importante et diversifiée à Paris. Différents enjeux selon ces acteurs se posent alors.

Les maternités parisiennes sont de plus en plus poussées à développer des réseaux professionnels pour améliorer la prise en charge des grossesses à risques et assurer le suivi prénatal des grossesses en ville. Bien que persiste encore aujourd'hui une certaine méconnaissance de la part des maternités de ce qui se passe autour d'elles, sous l'effet de pressions budgétaires et de différents leviers comme la contractualisation avec I'ARS, elles sont incitées à mieux connaître les ressources environnantes et développer des partenariats avec ces acteurs hors du soin.

La PMI de Paris compte 62 centres, ouverts à toutes les familles. Le principe d'universalisme proportionné et l'approche globale et préventive au fondement de ce service public conduisent la PMI à chercher à s'adapter aux besoins des familles, notamment des plus vulnérables. Des stratégies partenariales et $d^{\prime}$ « aller-vers » variées sont développées : déplacements à domicile ou dans certains foyers d'hébergement, groupes et ateliers dédiés à certaines populations, offre complémentaire à celle des maternités pour le suivi de grossesse des femmes très précaires ... La mise en place

des bébés parisiens sont nés de mère étrangère, soit deux fois plus que la moyenne nationale. 
de territoires de PMI, regroupant un ou plusieurs arrondissements chacun, doit permettre de constituer ces réseaux partenariaux au plus près du « terrain ».

Toutefois, faute de moyens humains suffisants, la PMI de Paris est amenée à suivre en priorité les familles les plus défavorisées (sans couverture sociale, vivant en centre d'hébergement ou en hôtel social, ...) et à rediriger les familles plus aisées vers l'offre libérale. Certains centres de PMI reçoivent ainsi en majorité des familles cumulant les vulnérabilités, ce qui rend l'accompagnement plus complexe. Il en résulte une tension entre la recherche permanente d'adaptation de la PMI et $\mathrm{I}^{\prime}$ « embolie » des services.

D'autres acteurs institutionnels ou de terrain interviennent en complément de ces deux acteurs principaux de l'offre de soins et de prévention.

L'ARS Ile-de-France finance directement différents réseaux de coordination : le réseau de santé périnatale parisien, d'autres réseaux régionaux thématiques couvrant Paris (femmes migrantes, nouveaux nés prématurés). Et dans le cadre du fond d'intervention régional, elle finance des projets « périnatalité et petite enfance », portés à Paris exclusivement par des associations soit situées dans un des quartiers prioritaires de la politique de la ville, soit travaillant auprès de publics en très grande précarité.

Des dispositifs comme les Ateliers Santé Ville (ASV) parisiens, implantés eux aussi dans les quartiers prioritaires de la politique de la ville, joue un rôle en œuvrant à la coordination d'acteurs sur le thème de la santé des femmes, notamment par la production d'annuaires, la mise en place de groupes de travail ou l'appui de projets locaux.

Enfin, des structures et des associations du champ social et médico-social, comme les centres sociaux, peuvent porter, seules ou conjointement, des projets pour améliorer l'accompagnement et la prise en charge des femmes et des familles. Le projet d'accompagnement de la maternité et à I'allaitement porté par Emmaüs Solidarité un projet, en partenariat avec la maternité de PortRoyal dans I'un de ses Espaces Insertion Famille en est un exemple (projet financé par I'ARS). Ces démarches ascendantes sont très souvent ancrées dans un territoire et partent de difficultés identifiées à cet endroit.

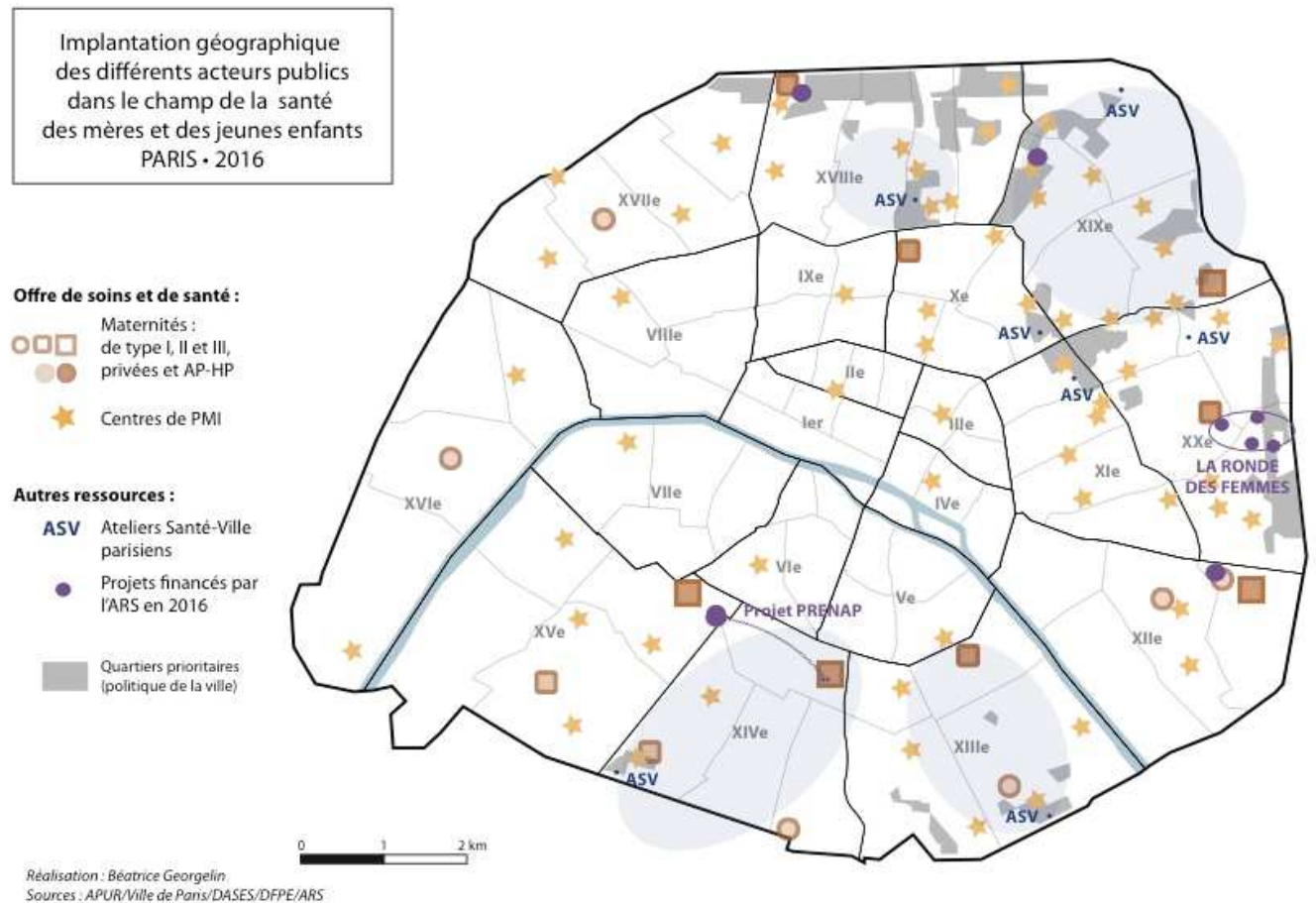

Carte 1. Implantation géographique des différents acteurs publics dans le champ de la santé des mères et des jeunes enfants à Paris mentionnés dans le résumé. 
3. Reconfigurations territoriales de l'action en santé : espaces, territoires et lieux

Plus que la réduction globale des inégalités, c'est l'amélioration de la prise en charge des personnes les plus précaires et les plus vulnérables qui est le moteur commun de dynamiques plurielles, opérant à différentes échelles.

Logiques de spatialisation des ressources. À l'échelle parisienne, tant l'ARS d'Ile-de-France que la Ville de Paris mettent en œuvre des politiques qui s'appuient sur des stratégies de spatialisation (Dubresson et Jaglin, 2005), veillant à ce que leurs ressources soient prioritairement allouées sur certains territoires parisiens, visant ainsi les populations les plus défavorisées. Les critères de priorisation territoriale pour l'attribution des crédits de l'ARS conduisent au ciblage exclusif de ces financements sur les quartiers prioritaires politique de la ville à Paris et/ou sur les personnes en situation de très grande exclusion. L'implantation géographique des centres de PMI et leur répartition sur Paris est perçue comme pertinente pour les directeurs de la PMI, les centres étant plus nombreux dans le nord-est parisien où la natalité est la plus forte et la part des familles vulnérables est la plus importante.

\section{Émergence et implantation de territoires} d'action. La forte densité d'acteurs sur Paris s'accompagne d'un manque de connaissance réciproque de ces acteurs, de leurs apports et de leurs fonctionnements. Les professionnels de terrain expriment fréquemment le besoin de davantage de mise en réseau et sont par ailleurs de plus en plus fortement enjoints à participer à des coordinations pluri-professionnelles. Ce besoin de coordination s'exprime à différentes échelles : tant au niveau parisien, qu'au niveau des arrondissements, qu'à un niveau plus local encore. Certains réseaux et projets locaux existants à Paris peuvent ainsi s'apparenter à de véritables «constructions locale de santé » (Fleuret, 2012), s'agissant de démarches portées par quelques acteurs implantés dans un territoire. Le projet «La Ronde des Femmes 》 dans le $20^{e}$, porté conjointement par plusieurs centres sociaux de l'arrondissement et en partenariat avec la maternité de l'hôpital Tenon, en est l'illustration : alors que certaines femmes venaient accoucher dans cette maternité sans trousseau, un projet associant plusieurs centres sociaux et leurs ressources propres s'est mis en place pour accompagner ces femmes dans la parentalité et l'accueil de l'enfant.

\section{Réflexion autour des lieux où favoriser} l'accès à l'offre de santé, notamment de prévention. Les réflexions autour de $l^{\prime}$ « allervers » et du décloisonnement de la santé appuient différentes initiatives: équipes mobiles, permanences de professionnels de santé dans les structures sociales, les accueils de jour, les centres d'hébergement, groupes de parole et accompagnement dans les structures associatives ... Une pluralité de lieux autres que les maternités et les centres de PMI peuvent être ainsi des lieux favorables à la santé et au bien-être des femmes enceintes.

Les modes d'actions possibles en ce sens, et les moyens disponibles pour, furent au cœur des débats des États Généraux de la PMI, un médecin de PMI ayant ainsi souligné la faiblesse de ces moyens : « La PMI hors les murs ? Mais il n'y a personne dans les murs!». Pour d'autres acteurs institutionnels se pose également la question du rapport coût/bénéfice, mesuré en terme de moyens humains alloués pour le nombre de personnes touchées par action, des actions menées dans des centres ou des foyers. Bien qu'elles permettent de toucher des personnes qui ne le sont pas par des campagnes de prévention universelles, elles sont beaucoup plus couteuses et donc fragiles, dans un contexte de réduction des effectifs et de diminution des enveloppes budgétaires.

Une certaine complémentarité entre ces stratégies apparaît, ainsi qu'une forte interdépendance des acteurs qui les portent, entre financeurs et opérateurs par exemple.

\section{Portée et limites : quelle offre de santé pour tous?}

La diversification des lieux et des approches, l'élaboration de projets locaux s'appuyant sur des dynamiques partenariales inscrites dans leur territoire, sont autant de leviers pour améliorer les parcours de santé de certaines femmes et familles, éloignées de la prise en charge proposée par un service public universel et généraliste.

Néanmoins, on peut souligner la concentration d'une part importante des ressources publiques sur certains quartiers et en direction de certains 
publics, les plus vulnérables. Cela soulève la question de la spécialisation de l'action publique et de l'impact que cela peut avoir sur les organisations professionnelles, notamment la PMI. Par ailleurs, on peut observer des effets pervers classiques quant aux zonages retenus pour conduire cette priorisation, qui prennent parfois difficilement en compte les territoires vécus par les habitants. Cela soulève également la question des moyens publics alloués pour la santé de toutes et des dynamiques à l'œuvre dans les autres territoires, non prioritaires. Enfin, face à des inégalités socio-territoriales de santé persistantes, ces stratégies sont-elles appelées à devenir des organisations définitives?

Par ailleurs, l'injonction à davantage de coordination ne résout pas toutes les difficultés posées par l'articulation de différentes approches en santé, d'univers distincts : santé publique, santé communautaire, travail social, $\cdots$

Les questions du financement et de la pérennité de ce système d'acteurs sont centrales, alors que l'ensemble des financements est à la baisse. Les acteurs non-institutionnels sont des relais indispensables de la politique de santé, mais on ne peut que constater la précarité de leur mode de financement, tant en volume disponible qu'en ce qui concerne les modalités, la temporalité et la prévisibilité de l'accès à ces financements.

En conclusion, deux enjeux principaux traversent cette présentation : les limites que rencontrent services publics et autres dispositifs institutionnels universalistes pour proposer une offre de santé adaptée à tous dans une métropole cosmopolite marquée d'importantes inégalités, et la fragilité du financement des acteurs associatifs, qui assurent pourtant aujourd'hui une part indispensable de l'offre de santé.

\section{Bibliographie}

BLONDEL B., KERMARREC M., 2011, « La situation périnatale en France en 2010, Premiers résultats de l'enquête nationale périnatale », Drees, Études et résultats, $n^{\circ} 775$, octobre 2011.

COHUET S., DANZON A., HAUSHERR E., ROGER S., 2015, « La santé des mères et des nouveaux nés, Données issues des premiers certificats des enfants nés en 2013 et domiciliés à Paris », DASES, Ville de Paris, en partenariat avec I'Atelier Parisien d'Urbanisme.

DUBRESSON A., JAGLIN S., 2005, « Gouvernance, régulation et territorialisation des espaces urbanisés: Approches et méthode », dans ANTHEAUME B., GIRAUT F. (dir.), Le territoire est mort, vive les territoires!: une (re)fabrication au nom du développement, Paris, France, IRD éd.

GASQUET C., 2013, « Itinéraires de santé liés au suivi et à l'issue de grossesse », étude réalisée la participation de $\mathrm{M}$. Collombier et d'A$C$ Hoyez, présentée à l'occasion des rencontres du réseau SOLIPAM en novembre 2013.

FLEURET S., 2012, Construction locale de santé. Attention primaire et santé communautaire, comparaisons internationales et expérimentation en France., Habilitation à diriger des recherches, Université d'Angers.

VANIER M., 2009, Territoires, territorialité, territorialisation: controverses et perspectives, Rennes, France, Presses Universitaires de Rennes (Entretiens de la Cité des Territoires), $228 \mathrm{p}$.

VINCELET C., LEBRETON E., POULAIN C., CRENN-HÉBERT C., MENGUY C., DANIEL AG., 2016, « La santé périnatale en Ile-de-France : Tableau de bord d'indicateurs départementaux et régionaux en périnatalité et orthogénie », mise à jour Mai 2016. Observatoire régional de santé Ile-de-France, ARS Ile-de-France.

Consultable en ligne à l'adresse suivante : www.perinat-ars-idf.org 


\section{UNE GEOGRAPHIE DES VULNERABILITES TERRITORIALES FACE AUX RISQUES ZOONOTIQUES EMERGENTS}

\section{Hélö̈se LUCACCIONI}

Laboratoire Dynamiques Sociales et Recomposition des Espaces (LADYSS), Université Paris Ouest Nanterre La Défense

Dans cette contribution, je présente mes travaux de thèse qui portent sur les dynamiques d'invasion d'un hôte de zoonose dans un territoire en mutation, à travers l'exemple du rat noir au Sénégal Oriental. J'y expose la problématique et les objectifs de cette recherche, le cadre théorique et méthodologique, puis les grandes étapes de la démonstration. À travers ces trois parties, je m'attache à montrer l'originalité de ma recherche et son insertion parmi les innovations et la variété des approches en géographie de la santé.

\section{Espaces, liens, et santé : problématique et objectif de la recherche}

Dans ma thèse de doctorat, je dresse une géographie des vulnérabilités territoriales face aux risques zoonotiques émergents. Cette recherche répond à des enjeux relatifs aux émergences infectieuses contemporaines face aux transformations des espaces et des sociétés, qui conjuguent changements globaux et intensification des relations spatiales (Armelagos 1988; Barrett et Armelagos 2013 ; Barrett et al 1998 ; Louria 2000 ; Mc Michael 2004 ; Morse 1995). En particulier, ma problématique renvoie à des préoccupations quant à l'expansion des aires de répartition d'espèces réservoirs et au risque associé d'émergence de zoonoses.

J'illustre cette problématique à travers les dynamiques d'invasion d'un hôte invasif, le rat noir (Aplin et al 2011; Battersby 2015; Meerburg et al 2009), dans une périphérie rurale en mutation d'Afrique de l'Ouest, le Sénégal Oriental.

Le positionnement du géographe sur cet objet a priori biomédical à l'interface des relations environnements-sociétés soulève un enjeu majeur : il s'agit de s'affranchir d'une approche centrée sur les mécanismes épidémiologiques et leurs facteurs de risque pour s'interroger sur le rôle des transformations des espaces et des sociétés dans les dynamiques spatiotemporelles du risque. En particulier, je m'interroge sur l'évolution des espaces et des liens et leur rôle dans les dynamiques d'invasion du rat noir. Je défends l'idée que les dynamiques (spatio-temporelles) du risque de zoonoses porté par le rongeur s'inscrivent dans des systèmes territoriaux complexes et multiscalaires, et je cherche à comprendre la construction, par les sociétés, de ces territoires vulnérables.

Cette recherche s'inscrit donc dans une problématique large qui croise le risque d'émergence infectieuse, les invasions biologiques, et les dynamiques territoriales à l'échelle du sud-est sénégalais. Au sein de cette problématique pluridisciplinaire, j'élabore des questionnements géographiques spécifiques: je souhaite analyser les interrelations entre les mises en liens et les transformations des espaces et des sociétés du sud-est sénégalais comme producteurs de vulnérabilité territoriale face à la diffusion spatiale du rat noir. Cette recherche revêt ainsi une dimension théorique et préventive face au risque de zoonoses portées par le rat noir.

2. Quelle géographie pour étudier les dynamiques spatio-temporelles du risque de zoonoses?

Cette recherche s'inscrit au croisement d'une géographie des maladies et d'une géographie nomothétique sur les processus de diffusion spatiale.

Avant tout, nos travaux s'approprient l'héritage d'une géographie des maladies d'inspiration francophone, dans la lignée des travaux de Max Sorre. Cette géographie sous-tend l'idée générale que la réunion de certaines conditions (biologiques, sociales, etc.) permet de délimiter l'espace du risque infectieux. Les travaux des géographes contemporains ont continué d'explorer les interrelations entre espaces et risque infectieux en se détachant du déterminisme du milieu et en développant une approche plus systémique. Ce sont les modes de gestion de l'environnement, les pratiques spatiales des sociétés, ainsi que les modes de contrôle et de gestion des territoires qui façonnent les disparités spatiales des faits 
infectieux (Hervouët et al 1995 ; Handschumacher 2011 ; Salem 1998).

En parallèle, les dynamiques spatio-temporelles du risque renvoient au «méga-concept » (Raffestin 1984) de la diffusion spatiale. Les travaux des géographes sur les phénomènes de diffusion s'inscrivent avant tout dans une géographie nomothétique, aux références majoritairement anglophones (par exemple, Pyle, Gould, Haggett, Cliff, etc.). Depuis les travaux précurseurs de Hägerstrand (1967), les géographes se sont efforcés d'élaborer un cadre théorique de ces processus spatio-temporels. En cherchant à relier l'organisation de l'espace des sociétés et les formes de la diffusion, ils mettent en évidence l'existence de régularités des formes spatiales de la diffusion et développent des modèles (vagues de diffusion, diffusion hiérarchique, diffusion par contagion, etc.).

Nous argumentons qu'un des enjeux principaux de la compréhension des phénomènes de diffusion est de réconcilier les formes spatiales et les processus sociaux explicatifs de ces dernières. Autrement dit, nous pensons que les phénomènes de diffusion spatiale révèlent l'organisation de l'espace au sens structurel (par exemple, la hiérarchie des lieux, leur connectivité, leur distance spatiale, etc.), mais également le fonctionnement des sociétés qui produisent cet espace (par exemple, par la diversité des processus sociaux, économiques, politiques, etc.).

En appliquant ce cadre méthodologique à notre problématique, nous faisons du rat noir un objet pour faire de la géographie : les dynamiques d'invasion du rongeur deviennent des marqueurs des dynamiques socio-spatiales. Ainsi, lire les vulnérabilités territoriales aux risques zoonotiques émergents portés par le rat noir en tant que phénomène de diffusion spatiale c'est avant tout chercher à comprendre comment les sociétés participent aux dynamiques spatio-temporelles d'invasion du rongeur.

3. Vulnérabilités territoriales au risque zoonotique émergent : quelques éléments de la démonstration

Notre recherche s'appuie sur le postulat selon lequel la progression du rat noir dépend essentiellement des transports offerts par I'homme. Au Sénégal, des travaux précédents
(Duplantier et al 1991 ; Konečný et al 2013) ont montré que la progression du rongeur depuis le XVème siècle s'est réalisée depuis les comptoirs coloniaux atlantiques jusqu'à l'intérieur des terres en s'appuyant sur les voies fluviales, les voies ferroviaires, et plus tardivement le réseau routier, supports des circulations de biens et de personnes.

Dans le Sud-Est du Sénégal, le rongeur atteint les marges de son aire de répartition. Il s'y étend à la faveur de nouvelles mises en liens à la fin des années 1990. En particulier, il est capturé pour la première fois dans l'extrême Sud un an après l'ouverture de la route bitumée qui relie les deux capitales régionales (Tambacounda et Kédougou) (Bâ 2002). Or le Sénégal Oriental est un territoire en mutation, une périphérie rurale qui s'ouvre sous l'influence de dynamiques historiques et contemporaines (attributions de diverses vocations agricoles, développement des corridors routiers internationaux, des activités extractives, etc.).

Pour comprendre le lien entre l'évolution des relations spatiales et les transformations des espaces, et les vulnérabilités territoriales à la diffusion du rongeur, notre recherche consiste en trois grandes étapes. Premièrement, nous dressons un diagnostic des dynamiques territoriales du Sénégal Oriental. Il s'agit d'une approche avant tout qualitative qui s'appuie sur la littérature scientifique et grise. Nous décrivons des espaces, des lieux, et des liens singuliers comme le produit d'héritages et de recompositions par les multiples opérateurs territoriaux : les disparités des espaces et des liens témoignent d'un encadrement territorial inégal selon les projets politiques et économiques des acteurs territoriaux successifs. En parallèle, nous réalisons un diagnostic de l'invasion du rongeur à partir de données d'archives et récentes de piégeages (Duplantier et al 2009). Nous montrons que la distribution du rat noir varie dans l'espace.

Dans une seconde étape, nous cherchons à établir une relation entre les variabilités spatiales et la distribution du rongeur. À l'aide de statistiques exploratoires, nous construisons des typologies des lieux à partir des caractéristiques locales susceptibles de conditionner leurs mises en liens (activités commerciales, connectivité au réseau routier et de transport, etc.). Pour cela, nous avons recours à des données primaires récoltées sur 
le terrain. Nous testons ensuite statistiquement des hypothèses sur la distribution du rat noir selon les types de lieux identifiés. Pour compléter cette analyse, nous dressons un portrait des mises en liens de ces lieux selon une approche qualitative. Autrement dit, nous explicitons la nature des relations spatiales (commerciales, agricoles, etc.) et leurs dynamiques selon les types de lieux.

Nos analyses aboutissent à un paradoxe : nous révélons une hiérarchie des lieux telle que les facteurs de vulnérabilité (caractéristiques de centralité, connectivité au réseau routier et de transport, etc.) apparaissent flous et complexes. En outre, ni cette hiérarchie des lieux, ni la distance spatiale ne permettent d'expliquer la diffusion du rongeur et sa répartition actuelle parmi les localités du Sénégal Oriental. En effet, le rat noir est présent là où on s'y attend le moins : dans les localités des marges agricoles les plus isolées au détriment des pôles commerciaux du goudron. Nous proposons alors de réinscrire la diffusion spatiale du rongeur en considérant non plus les lieux ou les liens seuls, mais les systèmes territoriaux.

Dans une dernière étape, nous réinterprétons la hiérarchie des lieux en dépassant les critères classiques d'analyse spatiale et des grilles de lecture structurelle des espaces. Nous proposons ainsi de comprendre les dynamiques d'invasion du rat noir dans l'articulation à plusieurs échelles des espaces, des lieux, et des liens. En cela, nous cherchons à réconcilier I'approche structurelle de l'organisation spatiale et le fonctionnement de ces territoires. Les systèmes territoriaux décrits apparaissent plus ou moins stables et porteur de différentes potentialités en matière de diffusion du rat. Combinés avec des systèmes bioécologiques, ils éclairent la répartition actuelle du rongeur dans les marges sud-est du Sénégal.

À travers des méthodes mixtes et un cadre méthodologique au croisement d'une posture constructiviste et nomothétique, nous dressons une géographie des vulnérabilités territoriales au risque zoonotique émergent incarné par le rat noir. En étudiant les dynamiques d'invasion du rat noir dans cette périphérie en transformation, je montre que nous ne sommes pas uniquement confrontés à des liens-supports mais à des systèmes sociaux et spatiaux qui articulent espaces, lieux, et liens.

\section{Bibliographie}

APLIN K.P., SUZUKI H., CHINEN A.A., CHESSER R.T., TEN HAVE J., DONNELLAN S.C., AUSTIN J., FROST A., GONZALES J.-P., HERBRETEAU V., CATZEFLIS F., SOUBRIER J., et al., 2011. Multiple Geographic Origins of Commensalism and Complex Dispersal History of Black Rats. PloS One. Vol. 6, $\mathrm{n}^{\circ} 11$, p. e26357. DOI 10.1371/journal.pone.0026357.

ARMELAGOS G.J., 1998. The Viral Superhighway. The Sciences. Vol. 38, $\mathrm{n}^{\circ} 1, \mathrm{p}$. 24-29. DOI 10.1002/j.23261951.1998.tb03354.x.

BÂ, 2002. Systématique, écologie et dynamique de populations de petits rongeurs potentiellement réservoirs ou hôtes de virus au Sénégal. Mémoire du diplôme de Sciences de la Vie et de la Terre de l'Ecole Pratique des Hautes Etudes. Montpellier, France : EPHE, IRD. 132 p.

BARRETT R., ARMELAGOS G., 2013. An Unnatural History of Emerging Infections. Oxford : Oxford University Press. 160 p. ISBN 978-0-19-960829-4.

BARRETT R., KUZAWA C.W., MC DADE T., ARMELAGOS G.J., 1998. Emerging and ReEmerging Infectious Diseases: The Third Epidemiologic Transition. Annual Review of Anthropology. Vol. 27, p. 247-271. DOI $10.2307 / 223371$.

BATTERSBY S.A., 2015. Rodents as carriers of disease. In BUCKLE A.P., SMITH R.H. (éd.), Rodent Pests and their Control. 2nd ed. Oxford : CABI. p. 81-100. ISBN 978-1-84593-817-8.

DUPLANTIER J.-M., GRANJON L., ADAM F., BA K., 1991. Répartition actuelle du rat noir (Rattus rattus) au Sénégal : facteurs historiques et écologiques. In LE BERRE M., LE GUELTE L. (éd.), Le rongeur et l'espace. Paris : $\mathrm{R}$. Chabaud. p. 339-346.

DUPLANTIER J.-M., GRANJON L., LE FUR J., PIRY S. (éd.), 2009. Base de Données sur les Rongeurs Sahélo-Soudaniens. CBGP, UMR (Inra/IRD/Cirad/Montpellier SupAgro).

HÄGERSTRAND T., 1967. Innovation Diffusion as a Spatial Process. Chicago, USA: University of Chicago Press. 334 p. ISBN 978-0-22631261-3. 
HERVOUËT J.-P., HANDSCHUMACHER P., LAFFLY D., 2004. Mobilités et espaces partagés au centre du risque sanitaire: l'exemple des endémies tropicales à transmission vectorielle. In GILBERT D. (éd.), Espaces tropicaux et risques : du local au global: Actes des 10èmes journées de géographie tropicale, 24-26 septembre 2003. Orléans, France : Presses Universitaires d'Orléans. p. 127-158.

HERVOUËT J.-P., HANDSCHUMACHER P., PARIS F., SALEM G., 1995. De la géographie des grandes endémies à la géographie de la santé et à la géographie tout court : vingt ans de travaux de I'ORSTOM en Afrique. Espace, populations, sociétés. Vol. $13, \mathrm{n}^{\circ} 1$, p. 59-65. DOI 10.3406/espos.1995.1671.

HANDSCHUMACHER P., 2011. Dynamique des espaces à risques sanitaires dans les pays du Sud : des systèmes entre tropicalité et maldéveloppement. In BART F. (éd.), Natures tropicales : enjeux actuels et perspectives. Bordeaux, France : Presses Universitaires Bordeaux. ISBN 978-286781729.

KONEČNÝ A., ESTOUP A., DUPLANTIER J.-M., BRYJA J., BA K., GALAN M., TATARD C., COSSON J.-F., 2013. Invasion genetics of the introduced black rat (Rattus rattus) in Senegal, West Africa. Molecular Ecology. Vol. 22, n², p. 286-300. DOI 10.1111/mec.12112.

LOURIA D.B., 2000. Emerging and re-emerging infections: the societal determinants. Futures. Vol. 32, n 6, p. 581-594.
MC MICHAEL A.J., 2004. Environmental and social influences on emerging infectious diseases: past, present and future. Philosophical Transactions of the Royal Society of London. Series B: Biological Sciences. Vol. $359, \mathrm{n}^{\circ}$ 1447, p. 1049-1058. DOI 10.1098/rstb.2004.1480.

MEERBURG B.G., SINGLETON G.R., KIJLSTRA A., 2009. Rodent-borne diseases and their risks for public health. Critical Reviews in Microbiology. Vol. 35, $\mathrm{n}^{\circ}$ 3, p. 221-270. DOI 10.1080/10408410902989837.

MORSE S.S., 1995. Factors in the emergence of infectious diseases. Emerging infectious diseases. Vol. 1, $\mathrm{n}^{\circ} 1$, p. 7-15. DOI 10.3201/eid0101.950102.

RAFFESTIN C., 1984. La diffusion. In BAILLY A. (éd.), Les concepts de la géographie humaine. Paris, France : Masson. p.189-193. ISBN 978-2225-80290-4.

SALEM G., 1998. La santé dans la ville : géographie d'un petit espace dense : Pikine (Sénégal). Paris, France: Karthala, ORSTOM. 360 p. ISBN 2-86537-826-8.

SORRE M., 1933. Complexes pathogènes et géographie médicale. Annales de Géographie. Vol. 42, $\mathrm{n}^{\circ}$ 235, p. 1-18. DOI 10.3406/geo.1933.10619. 
GENERATION D'ENVIRONNEMENTS POUR LA SIMULATION COMPORTEMENTALE DE VECTEURS EPIDEMIQUES : APPLICATION A AEDES AEGYPTI, VECTEUR DE LA DENGUE ET DU VIRUS ZIKA

\section{Renaud MISSLIN}

UMR IDEES 6266, Université de Rouen renaud.misslin1@univ-rouen.fr

Le moustique Aedes aegypti est le vecteur principal de la dengue et du virus Zika. Il est surtout présent dans les villes des zones tropicales et intertropicales d'Afrique, d'Amérique et d'Asie (Rodhain 1996). À moyen terme, les vaccins en cours de développement contribueront probablement au recul de ces maladies. Néanmoins, l'émergence ou la réémergence de maladies transmises par ces moustiques (comme la récente réémergence du Zika en Amérique Latine) font des mesures antivectorielles des éléments majeurs de la lutte contre ces maladies. Ces mesures consistent à modifier l'environnement dans le but de limiter le développement des moustiques (nettoyage, drainage et destruction des gîtes larvaires potentiels), de réduire leurs populations (traitements chimiques des gîtes, fumigation, introduction de prédateurs ou de mâles stériles) et de limiter les interactions entre hôtes et vecteurs (utilisation de moustiquaires ou de répulsifs). L'amélioration des moyens de surveillance des aires de distribution des vecteurs en milieu urbain et péri-urbain doit permettre d'optimiser l'utilisation de ces moyens dans le temps et l'espace (Gubler 2002).

Les facteurs qui influencent le développement de populations d'Ae. aegypti sont inégalement distribués dans les espaces urbains et leur présence évolue dans le temps. La variété de combinaisons de ces facteurs rend difficile la mise en évidence de causalités simples permettant d'expliquer et d'estimer les densités vectorielles. Les nombreuses études de terrain réalisées dans les villes d'Asie et d'Amérique permettent d'identifier des relations entre ces facteurs environnementaux et la présence de moustiques(e.g. Arunachalam et al. 2010). Cependant, ces observations empiriques valables au niveau local peuvent difficilement être transposées d'un contexte urbain à un autre. À titre d'exemple, la végétation est négativement corrélée à la présence de moustiques dans certaines villes (e.g. en Floride, États-Unis (Rey et al. 2006)) alors que dans d'autres cas, elle y est positivement corrélée (e.g. à Tucson, États-Unis (Landau et van Leeuwen 2012)). Les coûts élevés de ce type d'études de terrain sont susceptibles de les rendre inabordables pour de nombreuses administrations. Devant la difficulté de réaliser des mesures de cette diversité, de transposer les résultats de ces mesures et au vue de l'incapacité des expériences de laboratoire à reproduire les combinaisons de facteurs observées dans la réalité, la simulation informatique, plus particulièrement la simulation à base d'agents, apparait comme une alternative intéressante à ces obstacles. Cette méthode de modélisation permet de réaliser des expériences in silico sur des phénomènes difficilement observables dans la réalité.

Depuis 2008, plusieurs modèles à base d'agents permettent de reproduire les dynamiques spatiales des populations d'Ae. aegypti (e.g. Bomblies, Duchemin, et Eltahir 2008; Almeida et al. 2010; Karl et al. 2014). Quels que soient l'échelle et le terrain d'étude pour lesquelles ils sont produits, ces modèles présentent de notre point de vue un inconvénient majeur: ils ne sont pas transposables d'une ville à une autre, d'une période à une autre et d'une échelle à une autre. Dans le cadre du projet DENFREE (FP7), un modèle à base d'agents permettant de simuler les comportements de moustiques individuels dans l'espace a été implémenté (MOMA (Maneerat et Daudé 2016)). Cet outil permet d'étudier les dynamiques de population de moustiques évoluant dans des conditions environnementales spécifiques. Ce travail de thèse a pour objectif d'élaborer un modèle (MODE) permettant de reconstruire in silico des environnements urbains, aussi bien pour des agglomérations entières que des quartiers ou des ensembles de quartiers au sein de ces agglomérations. Afin de rendre le modèle transposable d'une ville à une autre ou d'une période d'étude à une autre, celui-ci est construit à partir de données libres (images satellites, données météorologiques) ou aisément accessibles (données démographiques intra-urbaines). 
L'objectif général de MODE est donc de reproduire les dynamiques spatiotemporelles des ressources et conditions permettant à Aedes aegypti d'accomplir son cycle de vie. Celles-ci sont définies dans MOMA qui simule le développement d'individus moustiques dont les besoins évoluent en fonction du stade de vie (3 stades aquatiques (œuf - larve - nymphe) et un stade adulte aérien). Les ressources sont au nombre de deux : le nectar et le sang. Elles sont consommées par les moustiques dans un objectif d'alimentation. Les conditions sont des facteurs environnementaux non-consommés influençant directement la répartition spatiotemporelle des moustiques. Il s'agit de l'eau fraiche et claire permettant aux moustiques d'accomplir leurs stades de vie aquatiques, des gites larvaires potentiels (récipient susceptibles de contenir de l'eau et de servir de lieu de ponte) et de la température de l'air, facteur essentiel de répartition aussi bien localement, à l'échelle de la ville (Misslin et al. 2016), qu'au niveau global (limite thermale aux latitudes $30^{\circ}$ Nord et Sud).
Les dynamiques spatiales et temporelles de ces ressources et conditions ne peuvent pas être mesurée directement par le biais d'images satellites ou de données démographiques librement disponibles (par exemple, les gites larvaires potentiels sont trop petits pour être visibles sur des images satellites (Moloney et al. 1998)). De ce fait, chacune de ces ressources et conditions ont été associées à des proxys traduisant la répartition des facteurs spatialement et temporellement (Figure 1). Ces distributions spatiotemporelles sont ensuite estimées pour une zone d'étude spécifique. Les proxys utilisés pour estimer les distributions des ressources sont la végétation, permettant d'estimer la distribution de nectar, et les hommes dont la densité permet d'estimer la capacité d'un espace à fournir du sang. Les répartitions spatiotemporelles des conditions sont estimées de façons similaires : la présence d'eau est liée aux précipitations, au remplissage manuel par des hommes et à l'évaporation et la présence de gites larvaires potentiels est liée au nombre de ménages (Karl et al. 2014). La dernière condition, la température de l'air, peut être estimée directement.

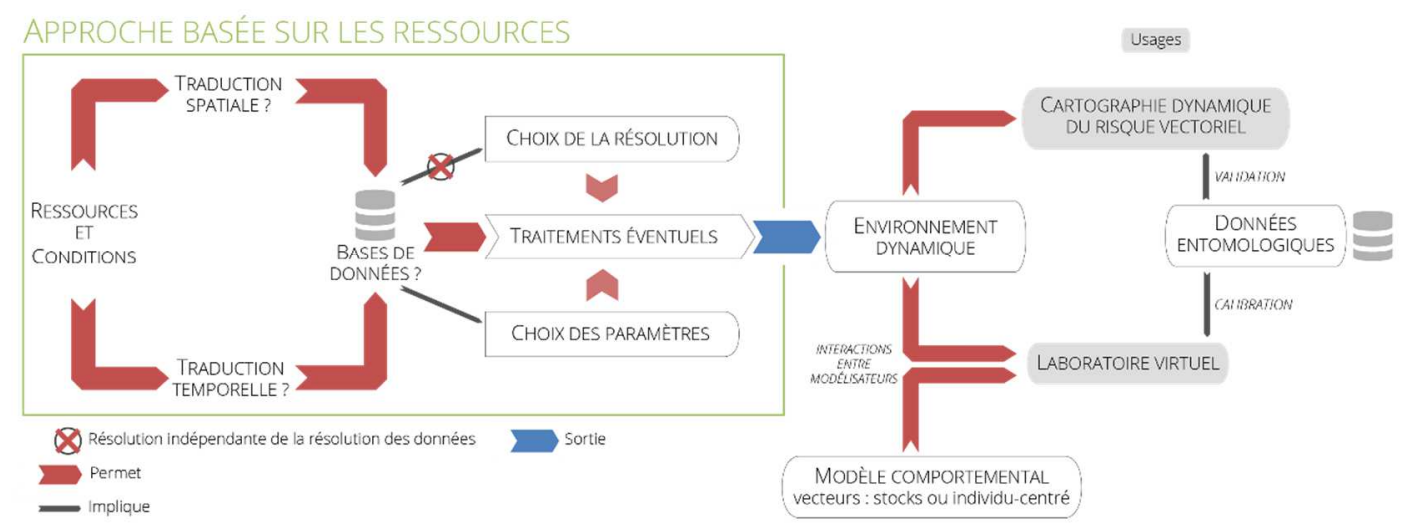

Figure 1 : Chaîne de traitements pour la construction de MODE 
MODE est donc un modèle d'environnement dynamique, implémenté sous GAMA, pouvant être couplé à MOMA. Un des objectifs de ce modèle est de permettre la génération d'environnements à plusieurs échelles différentes. Nous en avons identifié deux principales : celle de la ville dite «macro » et celle du quartier dite « micro ». L'échelle macro fait référence à une résolution de quelques dizaines (voir centaines) de mètres (typiquement : 30-50 m, zone correspondant approximativement à l'espace de vie d'un moustique) pour une étendue de plusieurs dizaines, voire centaines, de $\mathrm{km}^{2}$ (étendue qui correspond à la taille d'une grande ville. Cette échelle permet par exemple d'identifier les espaces les plus favorables aux moustiques dans une ville et guider les autorités sanitaires dans les opérations de lutte anti-vectorielle (Karl et al. 2014). Ce type d'environnement peut être associé à un modèle comportemental fonctionnant sous forme de stocks (la gestion d'individus moustiques à l'échelle d'une ville nécessiterait des capacités computationnelles bien trop élevées). L'échelle appelée « micro » dans le cadre de MODE est caractérisée par une étendue plus réduite (celle d'un bloc de bâtiments, d'un quartier) ne dépassant pas quelques $\mathrm{km}^{2}$. Sa résolution est de quelques mètres. Ce type d'environnement peut, une fois couplé avec un modèle individu-centré, être utilisé comme un laboratoire virtuel (Almeida et al. 2010).

À l'échelle macro, MODE se présente sous la forme d'une grille composée de «cellules environnement « (CE). Chaque $\mathrm{CE}$ est caractérisée par 5 variables correspondant aux ressources et aux conditions décrites ci-dessus. La génération de l'environnement à cette échelle est principalement basée sur le traitement d'images satellites OLI et TIRS (Landsat 8) et MODIS (Terra ou Aqua), sur des données démographiques spatialisées et sur des données météorologiques. La taille d'une CE est donnée par la résolution la plus fine parmi les images satellites utilisées, en l'occurrence, celle des images OLI et TIRS qui est de $30 \mathrm{~m}$. Le processus de génération de l'environnement consiste à donner des valeurs aux 5 variables contenues dans chacune des CE. La valeur de la variable ressource végétation est estimée grâce au Normalized Difference Vegetation Index (NDVI) (Tucker 1979) calculé sur des images OLI. Cet indice donne une mesure de l'activité végétale au sein d'un pixel. Le nombre d'habitants par CE est estimé par le biais d'une méthode de cartographie dasymétrique (Reibel et Agrawal 2007). Dans le cadre de MODE, cette méthode est utilisée pour affiner des données démographiques spatialisées en redistribuant la population par rapport à l'occupation du sol et la température de surface. De la même manière que les ressources, les conditions sont estimées par le biais d'un proxy. La présence d'eau est estimée par le biais de données de précipitations mesurées par une station météorologique localisée dans la ville étudiée. L'évaporation peut être calculée en se basant sur la température et la latitude grâce à l'équation de Linacre (Linacre 1977). La répartition des gites larvaires potentiels (GLP) dans l'espace urbain est très largement liée à la présence des hommes et à leurs activités (Ooi et al. 2006). Dans MODE, le nombre de GLP dans une CE est estimé en partant de l'hypothèse que les ménages en sont les principaux producteurs (les cellules environnement associées à de l'eau ne produisent pas de GLP). Le nombre de ménages par CE est calculé par une méthode de cartographie dasymétrique similaire à celle utilisée pour ventiler les individus. Enfin, l'estimation de la température de l'air est basée sur l'indice température-végétation (TVX) calculé sur des images MODIS (1 image toutes les 12h). L'approche TVX repose sur une hypothèse principale: la température de surface mesurée d'une canopée entièrement végétalisée est en équilibre thermique avec la température de l'air de cette canopée (Vancutsem et al. 2010). En partant de cette hypothèse, il est possible de calculer la relation entre la température de surface et la présence de végétation (mesurée par le NDVI). Si ces deux variables sont corrélées négativement, la méthode TVX peut être appliquée.

La génération d'environnements à l'échelle micro est entravée par la non-disponibilité de données libres et précises permettant de reconstruire la niche écologique potentielle du moustique. De fait, les environnements fins, comme ceux créés pour certains modèles à base d'agents individus-centrés permettant de simuler le comportement de moustiques (Almeida et al. 2010; Maneerat et Daudé 2016), incluent des données très fines, difficiles à extraire de façon directe d'images libres d'accès, souvent de moyenne résolution (e.g. OLI et TIRS). Afin de pallier cette limite, nous avons développé une méthode de génération « 
d'environnements synthétiques ». Celle-ci consiste à générer un environnement fin en se basant sur des données agrégées, de préférence libres, disponibles à une échelle plus large. La génération d'environnements synthétiques donne au modélisateur la possibilité de produire automatiquement des données réalistes répondant à ses attentes en termes d'échelle. Cette méthode présente un intérêt particulier pour les études menées dans des pays du Sud, sur des étendues relativement larges pour lesquelles les données fines d'occupation du sol (la localisation des bâtiments notamment) n'existent pas (même sur OSM) ou sont soumises à des accès restreints.

À l'échelle micro, MODE se présente sous la forme d'un ensemble d'objets vectoriels juxtaposés. La génération automatique de ces objets est basée sur les données produites à l'échelle macro. L'apport principal de cette démarche est sa capacité à produire des bâtiments individualisés. Ces bâtiments hébergent des habitants et ont un impact important sur la capacité de dispersion des populations de moustiques (Maneerat et Daudé 2016). La génération de l'environnement se déroule en plusieurs étapes successives. Le point de départ est une grille composée de «cellules environnements d dont les caractéristiques sont les mêmes que celles crées dans le cadre de la version macro de MODE. Dans un premier temps, chacune des cellules est définie comme «non-bâtie » ou «potentiellement bâtie » grâce à un tirage aléatoire pondéré par les indices de NDVI et de NDBI (Bhatti et Tripathi 2014) et en fonction de I'empreinte spatiale des réseaux routiers et hydrographiques issus d'Open Street Map. Ensuite, le nombre de ménages et le nombre d'habitants par CE sont utilisés pour constituer des agents «ménage » composés d'un ou plusieurs individus. Les bâtiments sont ensuite positionnés sur les CE en fonction du nombre de ménages correspondant à chacune des CE. Le nombre de GLP est calculé pour chaque bâtiment (GLP situés à l'intérieur) et pour chaque CE (GLP situés à l'extérieur). Enfin, la température de l'air calculée par le biais de la méthode TVX est affinée en fonction de la distribution des bâtiments. Les dynamiques de MODE-micro sont calquées sur les données météorologiques (précipitations, évaporation et température de l'air).
À chacune des échelles, MODE peut-être confronté à la réalité de terrain ou à d'autres bases de données dans un but de validation. À l'échelle macro, la cartographie dynamique du risque vectoriel émanant de l'assemblage des différentes caractéristiques des CE peut être comparée à des données vectorielles mesurées sur le terrain. À l'échelle micro, la validation peut être réalisée en comparant l'occupation du sol générée de façon synthétique à une occupation du sol issue de traitements réalisés sur des images de très haute résolution spatiale (e.g. Ikonos, Worldview 3, Quickbird). Une autre méthode de validation consisterait à comparer l'occupation du sol générée à des bases de données géographiques vectorielle produites par les administrations de la ville étudiée (e.g. BDTOPO de I'TGN). Le niveau de difficulté d'accès à ce type de base de données dépend du contexte local.

Les méthodes décrites ci-dessus peuvent être appliquées à de nombreuses villes situées dans les zones infestées par le moustique. La méthode proposée de génération d'environnements synthétique permet au modélisateur de s'affranchir en partie de la résolution des données initiales (Figure 1). À l'échelle macro aussi bien qu'à l'échelle micro, MODE permet de générer un environnement dynamique construit sous la forme d'un modèle à base d'agents. Ce modèle peut être utilisé pour déterminer la niche écologique potentielle du vecteur ou comme laboratoire virtuel s'il est couplé à un modèle à base d'agents permettant de simuler le comportement et les déplacements d'Ae. aegypti. Dans le dernier cas, le modèle résultat du couplage d'un modèle environnemental (MODE) et d'un modèle comportemental (MOM $A$ ) peut être calibré par le biais de mesures vectorielles effectuées sur le terrain. Cette communication consistera à présenter une application de la méthode à la ville de Bangkok (Thaïlande).

\section{Bibliographie}

Almeida, Sandro Jerônimo de, Ricardo Poley Martins Ferreira, Álvaro E. Eiras, Robin P. Obermayr, et Martin Geier. 2010. « Multi-Agent Modeling and Simulation of an Aedes Aegypti Mosquito Population ». Environmental Modelling \& Software 25 (12): 1490-1507. doi:10.1016/j.envsoft.2010.04.021. 
Arunachalam, Natarajan, Susilowati Tana, Fe Espino, Pattamaporn Kittayapong, Wimal Abeyewickreme, Khin Thet Wai, Brij Kishore Tyagi, Axel Kroeger, Johannes Sommerfeld, et Max Petzold. 2010. «Eco-bio-social determinants of dengue vector breeding: a multicountry study in urban and periurban Asia ». Bulletin of the World Health Organization $88 \quad$ (3): 173-84. doi:10.2471/BLT.09.067892.

Bhatti, Saad Saleem, et Nitin Kumar Tripathi. 2014. « Built-up Area Extraction Using Landsat 8 OLI Imagery ». GIScience \& Remote Sensing 51

445-67.

doi:10.1080/15481603.2014.939539.

Bomblies, Arne, Jean-Bernard Duchemin, et Elfatih A. B. Eltahir. 2008. « Hydrology of Malaria: Model Development and Application to a Sahelian Village: HYDROLOGY OF MALARIA ». Water Resources Research 44 (12): n/a-n/a. doi:10.1029/2008WR006917.

Gubler, Duane J. $2002 . \quad$ «Epidemic dengue/dengue hemorrhagic fever as a public health, social and economic problem in the 21st century ». TRENDS in Microbiology 10 (2): 100 $-103$.

Karl, Stephan, Nilimesh Halder, Joel K Kelso, Scott A Ritchie, et George J Milne. 2014. «A Spatial Simulation Model for Dengue Virus Infection in Urban Areas ». BMC Infectious Diseases 14 (1): 447. doi:10.1186/1471-233414-447.

Landau, Katheryn I., et Willem JD van Leeuwen. 2012. «Fine scale spatial urban land cover factors associated with adult mosquito abundance and risk in Tucson, Arizona ». Journal of Vector Ecology 37 (2): 407-418.

Linacre, Edward. 1977. «A simple formula for estimating evaporation rates in various climates, using temperature data alone ». Agricultural Meteorology 18: 409-24.

Maneerat, Somsakun, et Eric Daudé. 2016. « A Spatial Agent-Based Simulation Model of the Dengue Vector Aedes Aegypti to Explore Its Population Dynamics in Urban Areas ». Ecological Modelling 333 (août): 66-78. doi:10.1016/j.ecolmodel.2016.04.012.
Misslin, Renaud, Olivier Telle, Eric Daudé, Alain Vaguet, et Richard E. Paul. 2016. «Urban Climate versus Global Climate Change-What Makes the Difference for Dengue?: Climate, Dengue, and Urban Heat Islands ». Annals of the New York Academy of Sciences 1382 (1): 56 -72. doi:10.1111/nyas.13084.

Moloney, James M., Chris Skelly, Philip Weinstein, Maxine Maguire, et Scott Ritchie. 1998. «Domestic Aedes aegypti breeding site surveillance: limitations of remote sensing as a predictive surveillance tool. » The American journal of tropical medicine and hygiene 59 (2): 261-264.

Ooi, Eng-Eong, Kee-Tai Goh, Duane J. Gubler, et others. 2006. «Dengue prevention and 35 years of vector control in Singapore ». Emerg Infect Dis 12 (6): 887-893.

Reibel, Michael, et Aditya Agrawal. 2007. «Areal Interpolation of Population Counts Using Pre-Classified Land Cover Data ». Population Research and Policy Review 26 (56): 619-33. doi:10.1007/s11113-007-9050-9.

Rey, Jorge R., Naoya Nishimura, Billi Wagner, Marieta AH Braks, Sheila M. O'Connell, et L. Philip Lounibos. 2006. « Habitat segregation of mosquito arbovirus vectors in south Florida ». Journal of Medical entomology 43 (6): 11341141.

Rodhain, Francois. 1996. «Les insectes ne connaissent pas nos frontières ». Médecine et maladies infectieuses, $\mathrm{n}^{\circ} 26$ : 408-14.

Tucker, Compton. 1979. «Red and photographic infrared linear combinations for monitoring vegetation.pdf ». Remote Sensing of Environment 8: 127-50.

Vancutsem, Christelle, Pietro Ceccato, Tufa Dinku, et Stephen J. Connor. 2010. «Evaluation of MODIS Land Surface Temperature Data to Estimate Air Temperature in Different Ecosystems over Africa ». Remote Sensing of Environment $114 \quad$ (2): 449-65. doi:10.1016/j.rse.2009.10.002. 